\title{
La vitesse de défilement des barres de progression influence-t-elle la perception du temps d'attente?
}

\author{
Guillaume Gronier, Carine Lallemand \\ Centre de Recherche Public Henri Tudor \\ 29, avenue J.F. Kennedy \\ L-1855 Luxembourg \\ Tél : +352 425991856 \\ \{prénom.nom\}@tudor.lu
}

\begin{abstract}
RESUME
Cette recherche s'intéresse à la satisfaction et au temps d'attente perçu par l'utilisateur au cours des périodes où son interaction avec le système est momentanément suspendue (chargement d'un fichier, installation d'une application, etc.). Ces temps d'attente, souvent sources d'anxiété et d'agacement, sont généralement agrémentés par la présentation d'une barre de progression. L'objectif de cette recherche est d'étudier l'impact sur l'utilisateur des variations du défilement de ces barres de progression. Ainsi, nous nous intéressons plus particulièrement aux effets d'accélération et de décélération de leur remplissage. Les résultats confirment l'existence d'un lien causal entre perception du temps d'attente et satisfaction. De plus, une barre de progression qui décélère est significativement plus appréciée des utilisateurs. Les résultats obtenus pourront servir de recommandations pour la conception des interfaces.
\end{abstract}

\section{Mots clés}

Barre de progression, perception du temps d'attente, satisfaction, interfaces, métaphores.

\begin{abstract}
This research focuses on the satisfaction and waiting times perceived by a user during the moments where the interaction between the user and the system is temporarily interrupted (file download, setup of a program, etc.). These waiting times are often sources of anxiety and irritation. They go usually with the presentation of an animated progress bar. The objective of this research is to study the impact of different variable-rate progress bars by varying the speed of scrolling. Thus, we are interested in the effects of acceleration and deceleration between progress bars positions. The results confirm the existence of a causal link between perception of waiting time and satisfaction. In addition, a progress bar with a decelerating speed is significantly more appreciated by the users. The results may provide valuable information for the design of computer interfaces.
\end{abstract}

Permission to make digital or hard copies of all or part of this work for personal or classroom use is granted without fee provided that copies are not made or distributed for profit or commercial advantage and that copies bear this notice and the full citation on the first page. To copy otherwise, or republish, to post on servers or to redistribute to lists, requires prior specific permission and/or a fee.

$I H M^{\prime} 11$, October 24-27, 2011, Sophia Antipolis, France

Copyright (C) 2011 ACM 978-1-4503-0822-9/11/10 ...\$10.00.

\section{Categories and Subject Descriptors}

H.5.2 [Information interfaces and Presentation (e.g., HCI)]: User Interfaces---Graphical user interfaces (GUI).

\section{General Terms}

Human Factors, Experimentation.

\section{Keywords}

Progress bar, perception of waiting time, satisfaction, interfaces, metaphors.

\section{INTRODUCTION}

Le concept du temps qui passe a toujours fait l'objet de nombreuses réflexions chez l'homme. Aristote soulignait que la conscience temporelle ne saisit pas seulement le présent, mais aussi le passé et le futur immédiat. Au quotidien, lors des Interactions Homme-Machine (IHM), il n'est pas rare que l'utilisateur soit contraint d'attendre devant son ordinateur : le temps que son ordinateur charge une page internet, qu'il installe un logiciel ou encore qu'il démarre. En ergonomie, la plupart des recommandations pour l'utilisabilité des systèmes soulignent l'importance du feedback apporté à l'utilisateur sur l'état du système [20], notamment pendant ces périodes d'attente. Cependant, les recherches menées sur l'estimation des durées d'attente montrent que le fait qu'un utilisateur se concentre sur ces informations temporelles lui donne l'impression d'attendre plus longtemps [16]. D'où l'intérêt d'étudier l'impact des métaphores temporelles sur la satisfaction et le temps d'attente perçu des utilisateurs. Une première série d'expérimentations à caractère exploratoire [10] a montré que les barres de progression étaient les métaphores temporelles qui satisfaisaient le plus l'utilisateur, et lui donnaient le sentiment d'attendre moins longtemps en comparaison à d'autres métaphores (icône, texte, compteurs, page blanche). Dès lors, nous avons souhaité poursuivre notre compréhension de la perception du temps d'attente en étudiant l'impact des variations de la vitesse de progression. En effet, si les barres de progression sont fréquemment utilisées pour renseigner l'utilisateur d'un processus en cours, leur vitesse de remplissage peut accélérer ou décélérer.

\section{MODELES COGNITIFS DE LA PERCEPTION DU TEMPS}

En accord avec Kum, Lee et Yeung [14], nous considérons que la perception du temps d'attente n'est pas une construction cognitive linéaire et stable, dont la progression est estimée à partir de flux constants et continus. Elle repose au contraire sur des processus cognitifs complexes, qui 
tiennent compte à la fois du moment où l'estimation du temps perçu a lieu (évaluation pendant ou après le temps d'attente), mais aussi, lors d'une évaluation a posteriori, des mécanismes de récupération en mémoire dans lesquels interviennent les effets de récence et de primauté [21].

De plus, la perception du temps d'attente varie selon le contexte dans lequel elle s'inscrit [15] et de la tâche principale durant laquelle l'attente est imposée.

\subsection{Jugement Prospectif Versus Rétro- spectif}

Les recherches menées sur la perception du temps distinguent deux paradigmes en fonction du moment où l'évaluation de la durée perçue est réalisée par un sujet. On parle de jugements prospectifs quand les individus sont informés qu'ils vont devoir estimer la durée d'un intervalle de temps donné (durées expérimentées) et de jugement rétrospectif quand ils ne sont pas prévenus à l'avance (durées rappelées) [22]. Dans une méta-analyse réalisée sur 117 expérimentations, Block, Hancock et Zakay [2] montrent que quand la charge cognitive est élevée, l'évaluation prospective du temps diminue tandis que l'évaluation rétrospective augmente. L'évaluation prospective serait alors dépendante des processus attentionnels tandis que l'évaluation rétrospective serait influencée par les processus mémoriels.

Zakay [22] ajoute que dans une situation d'attente, un sujet devient automatiquement occupé par un jugement temporel prospectif car la durée d'attente attire son attention et devient le facteur le plus saillant.

\subsection{Effets de Primauté et de Récence}

Les recherches sur la mémoire ont mis en évidence le fait que les humains ne se remémorent pas les évènements de manière constante et linéaire, mais plutôt de manière sélective et biaisée ([1], [3]). Ainsi, les effets de primauté et de récence désignent respectivement le fait de se remémorer plus facilement des premiers ou des derniers instants d'un évènement. Contrairement à l'effet de primauté qui est stocké dans la mémoire à long terme (MLT), l'effet de récence dépend de la mémoire à court terme (MCT). Il est donc moins stable et peut être affecté par un intervalle de rétention dépassant les 15 à 30 secondes ou par la réalisation d'une activité interférente [8]. Une évaluation rétrospective du temps passé un tel délai ou suivant une activité interférente serait donc uniquement dépendante de l'effet de primauté. Une évaluation prospective ou rétrospective réalisée sans délai de rétention serait quant à elle dépendante à la fois de l'effet de primauté et de l'effet de récence.

\subsection{Le Modèle de la Porte Attentionnelle}

L'un des plus célèbres modèles cognitifs de la perception du temps est le modèle de la porte attentionnelle (MDA) du temps prospectif [23].

La première composante de ce modèle simplifié [4] est un pacemaker interne qui produit des impulsions dont le rythme est uniquement influencé par le niveau de stimulation : plus la personne est stimulée, plus le pacemaker produit de pulsations. La seconde partie du modèle se présente comme une porte qui s'ouvre plus ou moins largement en fonction de l'attention portée aux signaux temporels. Ouverte, elle laisse passer plus d'impulsions qui seront comptabilisées et relayées à la mémoire de travail. Plus une personne porte attention aux signaux temporels et plus la porte s'ouvre, laissant ainsi passer un grand nombre d'impulsions. A l'inverse, si la personne ne porte pas attention aux stimuli temporels ou qu'elle est distraite par d'autres évènements, la porte attentionnelle tendra donc à se refermer, ne laissant ainsi passer que peu d'impulsions. Dernier élément de ce modèle, la mémoire de travail va quant à elle créer une représentation du temps passé, basée essentiellement sur le nombre d'impulsions relayées. Suivant ce modèle, si on a la volonté de réduire la durée de temps perçue, il faut soit réduire le niveau de stimulation de la personne soit détourner son attention des signaux temporels [4] (Figure 1).

口

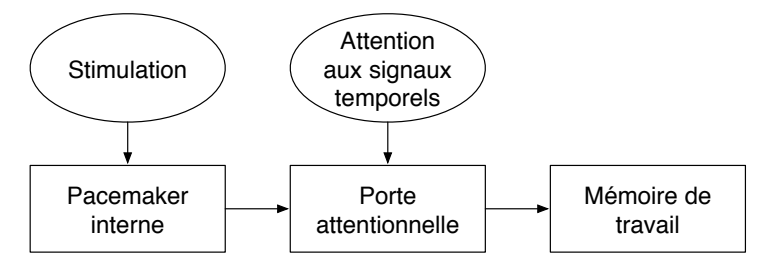

Figure 1 : Modèle de la porte attentionnelle de l'évaluation prospective du temps ([4], adapté de [23]).

\section{LE TEMPS D'ATTENTE DANS LES IHM}

\subsection{Le temps d'attente acceptable pour l'utilisateur}

Les premières études sur les délais d'attente acceptables pour l'utilisateur se sont rapidement accordées sur l'identification d'un seuil proche de 10 secondes. Nielsen [19] a, par exemple, identifié un palier de 10 secondes au-dessus duquel l'utilisateur ne se concentre plus sur sa tâche. Dans une étude sur la tolérance des utilisateurs face à une situation d'attente sur internet, Bouch [3] a recueilli les avis des utilisateurs sur les délais qu'ils jugeaient acceptables. En accord avec Nielsen [19], Bouch [3] a démontré qu'un délai supérieur à 10 secondes était considéré comme insatisfaisant, pouvait être source d'erreur pour l'utilisateur et réduire son efficacité au travail.

\subsection{Temps d'Attente et Retour d'Infor- mation}

Il est désormais largement admis qu'un retour d'information (feedback) sur la durée d'attente améliore l'utilisabilité d'une IHM ([19], [20]). Ce feedback peut prendre plusieurs formes : icône, barre de progression, message textuel, etc. Dans une étude sur le temps d'attente tolérable, [18] s'est intéressée à l'influence que peut avoir un feedback sur la satisfaction. L'auteur, en accord avec Geelhoed et al. [9] ou Bouch [3], a montré que la présence d'un feedback augmente considérablement le temps pendant lequel un utilisateur est prêt à attendre. Le feedback n'améliore pas seulement la confiance de l'utilisateur vis-à-vis du système, mais il le distrait pour mieux le faire patienter.

La tentation serait donc d'offrir à l'utilisateur un feedback très détaillé en le renseignant notamment sur la durée de l'attente et les détails du processus en cours. Cependant, si l'on peut penser naturellement qu'un feedback très informatif aidera à faire attendre l'utilisateur de manière plus efficace, il semble que le fait de donner beaucoup de détails sur l'avancement du processus en cours fait paraître l'attente plus longue [16]. En 
effet, le volume d'informations que l'utilisateur encode en attendant augmente la perception du temps d'attente. Comme il interprète chaque évènement comme coûteux en temps, l'utilisateur a l'impression qu'une période d'attente avec plus d'évènements prend plus de temps qu'une phase d'attente comprenant moins d'évènements [4]. Ces constats sont en adéquation avec le modèle de la porte attentionnelle décrit cidessus [4] puisque le côté très détaillé du feedback tend à focaliser l'attention de la personne sur les signaux temporels, ouvrant ainsi la porte attentionnelle et laissant passage libre à de nombreuses impulsions. Ces dernières seront comptabilisées par la mémoire de travail, qui évaluera ainsi le temps d'attente comme relativement long.

\subsection{Les Différentes Métaphores Tempo- relles}

Les barres de progression [17] sont souvent utilisées comme métaphore temporelle pour indiquer à l'utilisateur qu'un processus est en cours. Elles sont généralement représentées comme des barres se remplissant progressivement de $0 \%$ à $100 \%$ de la réalisation de la tâche en cours. Des études ont montré que, parmi les différents types de feedback donnés à l'utilisateur, les barres de progression obtiennent les meilleurs résultats, à la fois en termes d'acceptabilité de l'attention que de préférence des utilisateurs ([4], [11], [13]). Myers [17] montre que la présence d'une barre de progression pendant un temps d'attente améliore le sentiment d'efficacité et d'attractivité pour l'utilisateur. Grâce à la barre de progression, l'utilisateur peut savoir que sa requête a été prise en compte, qu'elle a été acceptée et interprétée, et que le système tente de lui donner une réponse. L'absence de barre de progression est source d'ennui, de doute et de déconcentration.

Une des limites inhérente à cette approche est qu'il est souvent difficile de déterminer précisément combien de temps l'attente de l'utilisateur va durer. Le processus de remplissage de la barre de progression est donc souvent variable, ce qui réduit la valeur informationnelle de cette dernière [4]. Désireuse de comprendre les effets de cette variabilité sur différents facteurs impliquant l'utilisateur, des études ont été menés pour mesurer l'influence de la vitesse de défilement sur la perception du temps d'attente ([11], [5]).

\subsection{Effets du Défilement des Barres de Progression sur la Perception du Temps d'Attente}

La problématique du défilement des barres de progression a été étudiée de manière expérimentale sous forme de comparaison dichotomique entre barres de progression à taux de remplissage constant ou barres à taux de remplissage variable [5]. Dans leur étude expérimentale, Harrison, Amento, Kuznetsov et Bell [11] vont plus loin et comparent le temps perçu entre 9 barres de durée égale mais dont la vitesse et le style de défilement variaient ( 8 selon des fonctions non linéaires et 1 selon une fonction linéaire). Seules deux barres dont la progression accélérait ont été perçues comme plus rapides que la barre de progression linéaire. Dans le cadre d'une évaluation prospective, il semble donc que l'effet de récence soit primordial. Pour augmenter la satisfaction des utilisateurs, les auteurs préconisent la mise en place d'une barre constante du processus puis d'une augmentation rapide du défilement sur la fin. Cependant, au vu de la méthodologie mise en place, où les sujets devaient comparer deux barres de progression entre elles (présentées l'une après l'autre) sur une durée très courte ( $5 \mathrm{sec}$. environ), il semble que les résultats traduisent plutôt une perception de la rapidité d'un processus visuel que d'une durée d'attente estimée. Les auteurs soulignent que des recherches complémentaires sont nécessaires.

\section{PROBLEMATIQUE ET HYPOTHE- SES}

Sur la base des études menées en sciences cognitives et en IHM sur la perception du temps d'attente, nous pensons qu'il est possible d'agir sur la perception de l'utilisateur afin de lui donner le sentiment d'attendre moins longtemps qu'en réalité [10]. Aussi avons-nous souhaité agir sur la perception de la vitesse de défilement d'une barre de progression (accélération vs décélération). En combinant les théories sur l'effet de primauté et le modèle de la porte attentionnelle, nous posons deux hypothèses :

- $\quad$ H1 : l'utilisateur aura l'impression d'avoir attendu moins longtemps dans la situation où la barre de progression décélère que pour une barre dont le défilement accélère. En effet, dans le cas d'un jugement rétrospectif, l'effet de primauté va donner une première impression de rapidité tandis que la décélération de la progression permettra de faire baisser le niveau de stimulation de l'utilisateur et de décentrer son attention des signaux temporels pour refermer la porte attentionnelle.

- H2 : l'utilisateur sera plus satisfait par une barre de progression qui décélère que par une barre de progression qui accélère. Ainsi, l'effet de primauté, moins éphémère que l'effet de récence, est agréable puisqu'il donne un feedback témoignant d'un avancement conséquent dès les 5 premières secondes d'attente. De plus, la perception d'un temps d'attente plus court postulée dans notre hypothèse 1 devrait mener à une meilleure satisfaction.

\section{METHODOLOGIE}

Afin d'évaluer la perception des variations du défilement des barres de progression, nous avons conçu 3 barres distinctes de même durée d'affichage (10 secondes): une barre qui décélère, une barre qui accélère et une barre constante (Figure 2).

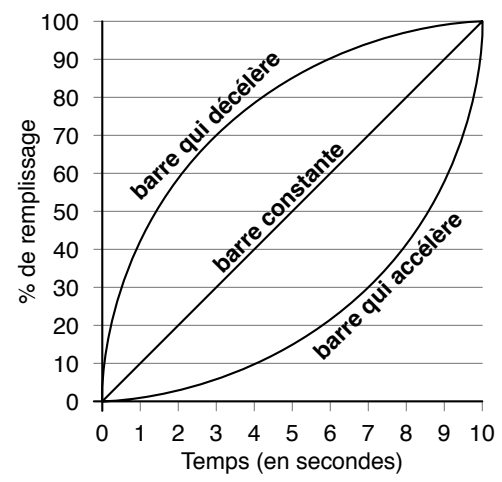

Figure 2 : Représentation graphique de la progression des trois différentes barres expérimentées.

Le remplissage des 3 barres de progression était calculé selon les formules suivantes : 
1. pour la barre dont le défilement s'accélérait, la progression était calculée sur base de la formule $f=\sqrt{\text { temps }} \times 3,16$;

2. pour la barre dont le défilement décélérait, la progression était calculée sur la base de la formule $f=$ temps $^{3} / 100$;

3. pour la barre au défilement constant, la progression était calculée sur la base de la formule $f=$ temps.

L'affichage à l'écran des barres de progression était assurée par une image animée (.GIF animé), qui donne l'assurance d'une compatibilité parfaite et d'une durée d'affichage constante entre toutes les plateformes (Microsoft Windows $\mathbb{C}$, Mac OS $\odot$, Linux $\odot$, etc.) et pour les principaux navigateurs récents (Internet Explorer, Firefox, Safari, Chrome, Opera, etc.). De plus, avant que l'expérience soit déployée auprès des utilisateurs, la durée d'affichage a été chronométrée et validée sur 10 configurations différentes : Firefox, Chrome et Safari sur Mac OS X ; Internet Explorer, Firefox, Safari et Chrome sur Windows 7 ; Internet Explorer et Firefox sur Windows XP ; Firefox sur Linux Ubuntu.

Pour tester nos hypothèses, nous avons développé un site Internet qui proposait, aux utilisateurs, un jeu de mémoire sollicitant l'exploration mentale d'une image [7]. Le jeu consistait à visualiser pendant 10 secondes une image fixe (la photographie d'un café parisien). Ensuite, l'utilisateur devait répondre à des questions-tests et se rappeler si la photographie préalablement affichée était composée ou non de 10 éléments (un vélo, une personne en terrasse, etc.). Ce jeu était un prétexte pour présenter à sa suite un écran d'attente de 10 secondes pendant l'étape d'enregistrement des réponses de l'utilisateur. Lors de cet écran d'attente était affichée l'une des 3 barres de progression, dont le choix était aléatoire. Enfin, 5 questions extraites et adaptées du Questionnaire for User Interaction Satisfaction (QUIS) [6] étaient posées à l'utilisateur afin de recueillir son niveau de satisfaction vis-àvis du site Web en général et du temps d'attente en particulier. Pour chaque question, une échelle de Lickert en 7 points a permis de recueillir un score de satisfaction global (tableau 1). Une analyse de fiabilité a confirmé le regroupement de ces 5 items, avec un alpha de Cronbach $(\alpha=0.823)$. L'alpha de Cronbach est un indice statistique variant entre 0 et 1 qui permet d'évaluer l'homogénéité d'un instrument d'évaluation composé par un ensemble d'items.

Tableau 1. Questionnaire de satisfaction extrait et adapté du QUIS [6]

\begin{tabular}{|c|c|c|c|c|c|c|}
\hline \multicolumn{5}{|c|}{$\begin{array}{l}\text { Le site Web est réactif... } \\
\text { pas d'accord }\end{array}$} & \multicolumn{2}{|c|}{ d'accord } \\
\hline 1 & 2 & 3 & 4 & 5 & 6 & 7 \\
\hline \multicolumn{7}{|c|}{$\begin{array}{l}\text { Le site Web tient informé des opérations en cours... } \\
\text { jamais }\end{array}$} \\
\hline 1 & 2 & 3 & 4 & 5 & 6 & 7 \\
\hline \multicolumn{7}{|c|}{ Dans l'ensemble, le site Web est... } \\
\hline lent & & & & & & apide \\
\hline 1 & 2 & 3 & 4 & 5 & 6 & 7 \\
\hline \multicolumn{7}{|c|}{ Le site Web est agréable à utiliser... } \\
\hline 1 & 2 & 3 & 4 & 5 & 6 & 7 \\
\hline \multicolumn{7}{|c|}{ Le temps d'attente avant la fenêtre récapitulative est... } \\
\hline long & & & & & & court \\
\hline 1 & 2 & 3 & 4 & 5 & 6 & 7 \\
\hline
\end{tabular}

Une dernière question ouverte demandait à l'utilisateur d'évaluer, en secondes, le temps qu'il estimait avoir attendu durant l'enregistrement des données : «Selon vous, combien de temps avez-vous dû attendre avant la fenêtre récapitulative? ». Par conséquent, l'évaluation du temps d'attente était rétrospectif [14].

\subsection{Population}

L'expérience, disponible en ligne, a été diffusée sur plusieurs canaux de communication (forums, réseaux sociaux, mailing list). 1127 sujets distincts (contrôle des adresses IP) y ont participé. L'âge moyen des sujets était de 24,9 ans $(\sigma=7,11)$, comprenant 105 femmes et 1022 hommes. La répartition des sujets selon le type de barre de progression est présentée dans le tableau 2.

Tableau 2. Descriptif de la population pour chaque barre de progression testée

\begin{tabular}{|l|c|c|c|c|}
\hline & Femme & Homme & Total & Age moyen \\
\hline $\begin{array}{l}\text { Barre qui } \\
\text { accélère }\end{array}$ & 37 & 355 & 392 & 24,4 ans \\
\hline $\begin{array}{l}\text { Barre qui } \\
\text { décélère }\end{array}$ & 31 & 345 & 376 & 24,6 ans \\
\hline $\begin{array}{l}\text { Barre } \\
\text { constante }\end{array}$ & 37 & 322 & 359 & 25,6 ans \\
\hline Total & $\mathbf{1 0 5}$ & $\mathbf{1 0 2 2}$ & $\mathbf{1 1 2 7}$ & $\mathbf{2 4 , 9}$ ans \\
\hline
\end{tabular}

\section{RESULTATS}

Les résultats de la perception du temps d'attente montrent qu'en moyenne les utilisateurs ont évalué leur temps d'attente à 8,57 secondes $(\sigma=4.07)$. Notons d'ailleurs que $27 \%$ d'entre eux ont trouvé le temps d'attente réel, qui était de 10 secondes.

En revanche, un test d'ANOVA, appliqué à la comparaison des moyennes pour chacune des barres de progression, montre qu'il n'existe pas de différence significative dans la perception du temps d'attente. Autrement dit, les utilisateurs ont eu le sentiment d'attendre la même durée quel que soit le type de barre de progression présentée. On ne constate pas non plus de différence significative entre l'évaluation du temps d'attente et nos variables sociodémographiques. Ni le sexe ni l'âge des utilisateurs n'ont joué un rôle particulier dans l'évaluation de cette durée.

Concernant les résultats relatifs à la satisfaction, de manière générale, on mesure une différence significative entre les 3 barres de progression $(F=3.003 ; p<.05)$. Il y a donc un effet de la variation de la vitesse de défilement des barres de progression sur la satisfaction des utilisateurs (Figure 3).

Plus précisément, on observe qu'il n'existe pas de différence significative entre la barre qui accélère et la barre constante, ainsi qu'entre la barre qui décélère et celle constante. En revanche, il existe une différence significative entre la barre qui accélère et la barre qui décélère, $(F=6,068 ; p<.05)$. Ainsi, la barre dont la vitesse de défilement ralentit au fur et à mesure de la progression est celle qui offre le plus de satisfaction. Au niveau des variables sociodémographiques, on note uniquement un effet significatif du sexe à la question 5 des items adaptés du QUIS. Ainsi, les femmes évaluent le temps d'attente sur une échelle de 1 à 7 comme un peu plus court que les hommes $(\mathrm{m}=4,63$ contre $\mathrm{m}=4,05$ pour les hommes). 


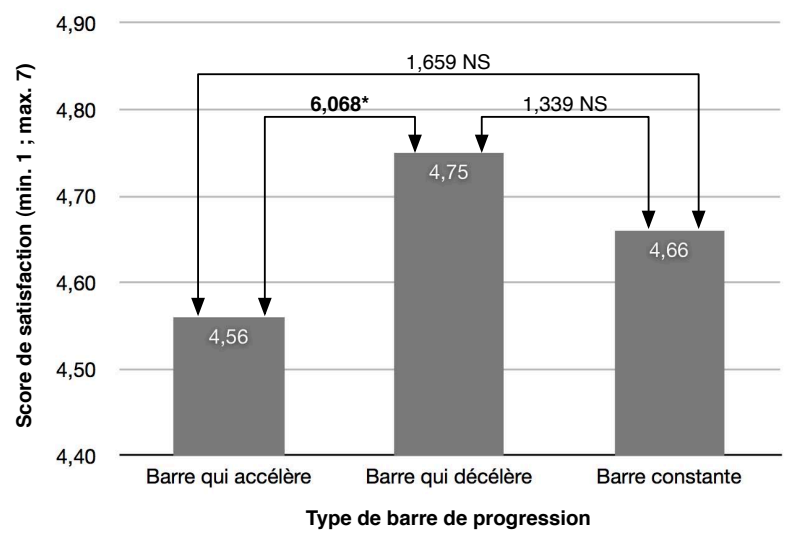

Figure 3 : Moyennes et comparaisons de moyennes (test ANOVA) de la satisfaction des utilisateurs selon le type de barre de progression (NS = Non Significatif).

Pour finir, soulignons qu'il existe une corrélation négative significative entre la perception du temps d'attente et la satisfaction $(r=-0,57, p<.05)$. Autrement dit, plus l'attente est perçue comme courte, plus les utilisateurs sont satisfaits.

\section{DISCUSSIONS}

Nos résultats confirment tout d'abord l'existence d'un lien causal entre perception du temps d'attente et satisfaction. Plus les utilisateurs ont trouvé le temps d'attente court et plus leur score de satisfaction a été élevé, et inversement. Cette observation vient appuyer les nombreuses études allant dans ce sens [19], [3], [18]

En revanche, nous n'avons pas mesuré de différence significative de la perception du temps d'attente entre les trois barres de progression. Par conséquent, notre première hypothèse (H1 : l'utilisateur aura le sentiment d'attendre moins longtemps pour une barre de progression qui décélère que pour une barre de progression qui accélère) n'est pas vérifiée. Plusieurs raisons peuvent être évoquées. Tout d'abord, la durée choisie de 10 secondes ne constitue peut-être pas une durée d'attente assez longue. En effet, étant égale au seuil raisonnable d'attente défini par Nielsen [19] et Bouch [3], elle ne provoque pas de déconcentration ni de frustration chez l'utilisateur. De plus, la variation de l'accélération ou de la décélération de la barre de progression a un impact moins visible sur une courte durée. Une étude faisait varier les durées d'attente comme celle de Branaghan et Sanchez [4] permettrait de voir si des différences significatives apparaissent pour des délais d'attente plus longs.

Nos résultats montrent que la barre qui décélère est significativement la plus appréciée des utilisateurs (Figure 2). Notre seconde hypothèse H2 (l'utilisateur sera plus satisfait par une barre de progression qui décélère que par une barre qui accélère) pour la satisfaction est confirmée. Les utilisateurs semblent donc bel et bien évaluer leur perception du temps sur le début du défilement de la barre de progression, répondant ainsi à un effet de primauté.

Ces résultats viennent corroborer les premières intuitions de Kum, Lee et Yeung [14]. Ces derniers proposant même de concilier totalement les divergences entre processus perceptifs et mnésiques en affichant des barres de progression «fast- slow-fast », c'est-à-dire qui accélèrent dans leur premier tiers de défilement, qui décélèrent dans leur second tiers, et qui accélèrent de nouveau dans leur troisième tiers. Ceci dit, s'il permettrait de combiner les effets positifs de primauté et de récence, ce paradigme risquerait de focaliser l'attention des utilisateurs sur le processus d'attente en leur donnant l'impression d'assister à trois «évènements » distincts se succédant [4].

Concernant le temps d'attente, les résultats montrent qu'il n'y a pas de différences significatives entre les 3 barres, même si la barre constante a été évaluée plus proche des 10 secondes réelles que les 2 autres barres. Ces résultats peuvent non seulement s'expliquer par la difficulté que rencontrent les utilisateurs à évaluer le temps qu'ils ont attendu, mais aussi par la nature de la tâche principale. Leclerc, Schmidt et Dubé [15] ont montré que l'évaluation du temps dépendait largement du contexte et des caractéristiques de la situation dans laquelle les sujets devaient faire part de leur évaluation. Si dans l'expérience de Leclerc et al. [15] les utilisateurs étaient placés dans des conditions où ils pouvaient gagner ou perdre de l'argent pendant leur attente (ce qui influençait leur perception du temps qui s'écoulait), leurs résultats ont montré de façon plus générale toute l'importance du contexte et de la nature de la tâche dans les processus perceptifs.

Concernant le fait que l'évaluation du temps soit plus proche de la réalité dans le cas d'une barre de progression constante, on peut dire que, si cette tendance se confirmait dans des études ultérieures, son explication pourrait être trouvée dans modèle de la porte attentionnelle de l'évaluation prospective $\mathrm{du}$ temps [5]. En effet, une barre de progression constante aurait un effet relativement neutre à la fois sur le processus de stimulation de l'utilisateur et sur l'attention portée aux signaux temporels. La barre constante pouvant être interprétée comme un seul évènement prévisible, les impulsions du pacemaker interne seraient alors transmises à un rythme constant et proche de la réalité à la mémoire de travail.

Notons également, pour notre expérimentation, que le temps évalué était toujours inférieur d'environ 1,5 secondes par rapport à la durée d'attente réelle $(M=8,57$ pour la barre qui accélère ; $M=8,76$ pour la barre constante ; $M=8,37$ pour la barre qui décélère), ce qui tend à démontrer que notre contexte, c'est-à-dire le jeu de mémorisation, favorisait une évaluation plus «optimiste» du temps d'attente. Contrairement à Harrison et al. [11] qui demandaient à chaque sujet de comparer deux barres de progression, nous avons en effet préféré un protocole de recherche où chaque barre était placée dans le contexte d'une tâche principale, comme c'est souvent le cas dans la réalité. Or, il est probable que les sujets n'aient pas accordé toute leur attention à l'attente qui leur était imposée. Ce constat est en adéquation avec le modèle de la porte attentionnelle [5]. Moins l'attention est portée sur les signaux cognitifs et moins les impulsions temporelles parviennent à la mémoire, donnant l'impression à l'utilisateur d'avoir attendu moins longtemps.

A ce sujet, certains auteurs [13] proposent, au-delà de l'amélioration des barres de progression en elles-mêmes, de réfléchir à la manière dont on peut aider l'utilisateur à utiliser au mieux ce temps d'attente. Les barres de progression actives qu'ils décrivent dans leur étude de 2011 offrent ainsi à l'utilisateur des fonctionnalités lui permettant de réaliser des activités temporaires durant l'attente (ex : consultation de leur agenda ou mails, lecture de leur liste de tâches à réaliser ou 
encore d'informations sur l'actualité ou la météo). Bien qu'il faille prendre garde au contrôle des actions par l'utilisateur dans ce contexte, il nous semble que ces travaux ouvrent de nouvelles perspectives en IHM.

\section{CONCLUSIONS ET PERSPECTIVES}

Nos résultats montrent, dans notre contexte d'expérimentation, qu'une barre de progression dont la vitesse décélère offre une plus grande satisfaction qu'une barre de progression constante ou qui barre dont le défilement s'accélère. Comme nous l'avons souligné précédemment, les conclusions de cette étude doivent tout d'abord être pondérées par la nature de la tâche principale durant laquelle est imposé le temps d'attente. Une étude complémentaire pourrait être menée afin de mesurer l'influence de différents contextes perceptifs. La durée relativement courte et acceptable de l'attente pourrait quant à elle expliquer l'absence de différence significative entre nos trois barres de progression en ce qui concerne la perception du temps d'attente. En accord avec la théorie des seuils de perception, les mouvements d'accélération et de décélération peuvent ne pas être suffisamment bien perçus lors d'attentes trop courtes (inférieures à $10 \mathrm{sec}$.).

D'autres travaux pourront être envisagés, notamment pour clarifier le rôle des éléments constitutifs du modèle de la porte attentionnelle, qui semble à même d'apporter des pistes de compréhension des phénomènes liés à la perception du temps d'attente. Ainsi, en plus de varier les délais d'attente, on pourrait introduire différents niveaux de stimulation des utilisateurs ainsi que des feedback de progression du processus plus ou moins informatifs (focalisant plus ou moins l'attention de l'utilisateur sur les signaux temporels). Dans cet état d'esprit, Harrison et al. [12], ont mené une étude sur les effets d'animation appliqués aux barres de progression. Ils ont montré par exemple que les barres de progression présentant un effet animé de style "nervures» - se déplaçant vers l'arrière en décélérant - ont permis de réduire la perception du temps d'attente de $11 \%$ au sein de leur échantillon.

De nombreuses pistes restent à explorer mais nous pouvons retenir que, comme pour toute activité relevant du domaine de l'ergonomie, la conception des barres de progression et autres métaphores du temps d'attente en IHM, doit faire l'objet de compromis. C'est une fois encore le contexte d'utilisation qui sera à même de déterminer les choix de conception afin d'assurer à l'utilisateur le meilleur confort d'utilisation possible.

\section{REMERCIEMENTS}

Les auteurs remercient et félicitent vivement les étudiants de la promotion 2011 de l'Institut Supérieur Franco-Allemand de Techniques, d'Economie et de Sciences (ISFATES) qui ont participé à cette expérience: C. Anthony, T. Billet, S. El Hadri, F. Klauss, G. Marillier, N. Mathieu, J. Meiser, A. Nkome Ndjebayi, S. Piedoux, R. Plasse, A. Tauraatua, A. Tavan, X. Vare, B.A. Yongueng Dihewou, A. Youmsi Waffo, M. Zéler.

Cet article est tout particulièrement dédié à la mémoire de Marion Zéler.

\section{BIBLIOGRAPHIE}

[1] Allan, L.G. The perception of time. Perception and Psychophysics 26, (1979), 340-354.
[2] Block, R.A., Hancock, P.A., and Zakay, D. Acta Psychologica How cognitive load affects duration judgments : A meta-analytic review. Acta Psychologica 134, 3 (2010), 330-343.

[3] Bouch, A., Kuchinsky, A., and Bhatti, N. Quality is in the eye of the beholder: meeting users' requirements for Internet quality of service. Proceedings of the SIGCHI conference on Human factors in computing systems, ACM (2000), 297-304.

[4] Branaghan, R.J. and Sanchez, C.A. Feedback preferences and impressions of waiting. Human Factors 51, 4 (2009), 528-538.

[5] Branaghan, R.J. and Sanchez, C.A. Feedback preferences and impressions of waiting. Human Factors 51, 4 (2009), 528-538.

[6] Chin, J.P., Diehl, V.A., and Norman, K.L. No Development of an instrument measuring user satisfaction of the human-computer interface. Proceedings of the SIGCHI Conference on HUman Factors in Computing Systems, USA, Washington, (1998), 213-218.

[7] Denis, M. and Kosslyn, S.M. Scanning visual mental images: A window on the mind. Current Psychology of Cognition 18, (1999), 409-465.

[8] Gaonac'h, D. and Larigauderie, P. Mémoire et fonctionnement cognitif : la mémoire de travail. Paris : Armand Collin, 2000.

[9] Geelhoed, E., Toft, P., Roberts, S., and Hyland, P. To influence time perception. Conference companion on Human factors in computing systems - CHI '95, ACM Press (1995), 272-273.

[10] Gronier, G. and Gomri, S. Etude des métaphores temporelles sur la perception du temps d'attente. 20ème Conférence Francophone sur l'Interaction HommeMachine, Metz, 2-5 Septembre, (2008), 205-208.

[11] Harrison, C., Amento, B., Kuznetsov, S., and Bell, R. Rethinking the progress bar. Proceedings of the 20th annual ACM symposium on User interface software and technology - UIST '07, ACM Press (2007).

[12] Harrison, C., Yeo, Z., and Hudson, S.E. Faster progress bars : manipulating perceived duration with visual augmentations. ACM CHI'2010, (2010).

[13] Hurter, C., Girouard, A., Riche, N., and Plaisant, C. Active progress bars : facilitating the switch to temporary activities. CHI 2011, May 7-12, Vancouver, $B C$, Canada, (2011), 1-6.

[14] Kum, D., Lee, Y.H., and Yeung, C. The Velocity of Time: Primacy and Recency Effects on Time Perception. Advances in Consumer Research 35, 1 (2007), 943-944.

[15] Leclerc, F., Schmitt, B.H., and Dube, L. Waiting Time and Decision Making: Is Time like Money? Journal of Consumer Research 22, 1 (1995), 110.

[16] Macar, F., Grondin, S., and Casini, L. Controlled attention sharing influences time estimation. Memory \& Cognition 22, (1994), 673-686. 
[17] Myers, B.A. The importance of percent-done progress indicators for computer-human interfaces. ACM CH'I85 Proceedings, (1985), 11-17.

[18] Nah, F.F.-hoon. A study on tolerable waiting time: how long are Web users willing to wait? Behaviour \& Information Technology 23, 3 (2004), 153-163.

[19] Nielsen, J. Usability Engineering. Morgan Kaufmann, San Francisco, 1994.

[20] Scapin, D. and Bastien, J.M.C. Ergonomic criteria for evaluating the ergonomic quality of interactive systems.
Behaviour \& Information Technology 16, 4 (1997), 220231.

[21] Soman, D. Prospective and retrospective evaluations of experiences: how you evaluate an experience depends on when you evaluate it. Journal of Behavioral Decision Making 16, 1 (2003), 35-52.

[22] Zakay, D. Attention et jugement temporel. Psychologie Française 50, 1 (2005), 65-79.

[23] Zakay, D. and Block, R.A. Temporal Cognition. Current Directions in Psychological Science 6, 1 (1997), 12-16. 